\title{
Review
}

\section{The Future of Brain Imaging in Parkinson's Disease}

\author{
Rick C. Helmich ${ }^{\mathrm{a}, *}$, David E. Vaillancourt ${ }^{\mathrm{b}}$ and David J. Brooks ${ }^{\mathrm{c}}$ \\ ${ }^{a}$ Radboud University Medical Centre, Donders Institute for Brain, Cognition and Behaviour, \\ Donders Centre for Cognitive Neuroimaging, Nijmegen, The Netherlands \\ ${ }^{\mathrm{b}}$ University of Florida, Applied Physiology and Kinesiology, Neurology, \\ and Biomedical Engineering, Gainesville, FL, USA \\ ${ }^{\mathrm{c}}$ Department of Nuclear Medicine and PET Centre, Aarhus University Hospital, Aarhus, Denmark, \\ Division of Neuroscience, Newcastle University, Newcastle, UK
}

Accepted 31 October 2018

\begin{abstract}
Parkinson's disease (PD) is a progressive neurodegenerative disorder that is associated with distinct abnormalities in brain function and structure. Here we discuss how future developments in functional, structural and nuclear brain imaging may help us to better understand, diagnose, and potentially even treat PD. These new horizons may be reached by developing tracers that specifically bind to alpha synuclein, by looking into different places in the body (such as the gut) or in smaller cerebral nuclei (with improved spatial resolution), and by developing new approaches for quantifying and interpreting altered dynamics in large-scale brain networks.
\end{abstract}

Keywords: Parkinson's disease, biomarkers, neuroimaging, magnetic resonance imaging, positron emission tomography

\section{INTRODUCTION}

Parkinson's disease (PD) is a progressive neurodegenerative disorder characterized by motor symptoms such as bradykinesia, rigidity and tremor, and a large variety of non-motor symptoms. The pathological hallmark of the disease is a profound loss of nigro-striatal dopamine cells and an accumulation of intracellular inclusions called Lewy bodies, which are comprised of alpha-synuclein aggregates. In concert with dopaminergic cell loss, several other neurotransmitter systems also degenerate, and in later stages Lewy body pathology also spreads to the cortex. This makes PD a prototypical systems disorder,

\footnotetext{
*Correspondence to: Rick Helmich, Radboud University Medical Centre, Donders Institute for Brain, Cognition and Behaviour, Donders Centre for Cognitive Neuroimaging, PO BOX 9101, 6500 HB Nijmegen, The Netherlands. E-mail: rick.helmich@donders.ru.nl.
}

which can be fully understood only when investigating molecular, structural and functional abnormalities at the level of brain networks $[1,2]$. With the rise of neuroscience in the last few decades, a plethora of new methods and approaches have become available. In this paper, we review and speculate how future developments in functional imaging, structural imaging, and nuclear imaging can help us understand, diagnose or even treat PD.

\section{FUNCTIONAL IMAGING}

Imaging of brain activity can be done with functional magnetic resonance imaging (fMRI) or positron emission tomography (PET). These approaches have made significant contributions to our understanding of the pathophysiology of PD. The field has grown from focussing on abnormal, 
task-related activity in isolated brain regions (such as the putamen) to demonstrating abnormal interactions between intrinsic, large-scale networks in patients with PD (such as the cortico-striatal circuit). For example, a recent paper has demonstrated that PD patients spend more time in "brain states" that are characterized by increased interactions between cerebral networks, at the expense of more sparsely connected, segregated brain states [3]. The shift from single brain regions to large-scale networks comes with new difficulties: the data have become multi-dimensional, and this makes it more difficult to extract meaningful patterns. The challenge is to extract biologically relevant parameters that capture (dys)function of an entire network.

An existing approach that has been used for a long time is the Parkinson's Disease Related Pattern (PDRP), i.e., a metabolic covariance pattern (or network) that is specific to PD, and that can be calculated using PET and fMRI data [2]. The validity of this approach has been proven in different centres across the world, although it has not been translated into standard clinical practice. This is probably because it works best with PET, which is not widely available. A second, more recently developed approach to deal with multi-dimensional data is the use of generative models, i.e., computational models that estimate how observed (fMRI) data were generated, given a set of priors and hypotheses regarding the configuration of the network [4]. This approach, which is implemented in dynamic causal modelling (DCM), has the advantage that different competing models of brain function can be compared within a group or between two groups (using Bayesian model selection). In PD, DCM has been used to test how network interactions give rise to tremor and to (abnormal) voluntary actions [5-7]. Inter-individual differences in statistical evidence for a particular model of brain function may be used to define (and clinically validate) imaging-based PD phenotypes, or even to support differential diagnosis through Bayesian model selection [4]. Finally, a third approach is to extract biologically meaningful features from multi-dimensional imaging data. For example, using resting state fMRI data, parameters can be calculated that reflect the gradient of corticostriatal connectivity across striatal subregions [8]. These parameters (such as the direction and steepness of the gradient) are biologically meaningful, because they reflect the underlying anatomy and distribution of dopamine. Also, they may be sensitive to changes in cortico-striatal connectivity, which are known to occur in PD [9]. These data could be used to build normative models (analogous to "growth curves") to determine whether an individual departs from the normal pattern [10]. If clinically validated, such approaches may be helpful for diagnosis and disease monitoring.

A second development could lie in improved functional MRI sequences, enabling brain imaging at a high temporal and/or spatial resolution. Although fMRI is thought to be too slow to measure brain oscillations, given that it depends on slow changes in blood flow, recent studies have used ultra-rapid fMRI scanning at 7 Tesla to measure visual cortex BOLD oscillations at $0.75 \mathrm{~Hz}$ following high-frequent visual stimulation [11]. A further study showed resting state connectivity at frequencies up to $2.5 \mathrm{~Hz}$ [12]. Although it remains to be seen whether these signals carry information that is different from the slower BOLD fluctuations, it is possible that future developments will allow us to measure pathological oscillations (e.g., tremor) with fMRI. Furthermore, imaging at both high spatial and temporal resolution makes it possible to detect abnormal fluctuations in small brain stem nuclei while adequately correcting for rapid non-neuronal noise (e.g., cardiac and respiratory fluctuations around the brain stem). Given that the pathophysiological hallmark of PD is a degeneration of several neurotransmitter systems in the brain stem, these new techniques make it possible to test for altered activity and functional connectivity of these nuclei.

\section{STRUCTURAL IMAGING}

Structural imaging in PD has made important advances over the past decade [13], and as we look to the future several important themes emerge. The first theme is high-field imaging and improved spatial resolution having a significant impact on visualizing and quantifying features of the basal ganglia and other regions going forward. We are beginning to see progress in this area already in that 7 Tesla structural imaging has enabled automated segmentation of the substantia nigra, subthalamic nucleus, and red nucleus, which is not available at 3 Tesla [14]. Using a multimodal protocol, in vivo mapping of the basal ganglia and thalamic connections was achieved at submillimetre resolution and included susceptibility weighted imaging, diffusion imaging, and T1 and T2 weighted imaging [15]. These advances in submillimetre structural imaging have not yet made their 
way to routine patient studies but have the potential to enhance clinical practices such as diagnosing PD and DBS surgery.

The second theme is in linking diffusion imaging as a biomarker of differential diagnosis, progression of PD, and relation to pathology. Recent evidence has linked diffusion imaging as a predictor of both cognitive function and motor symptoms in PD [16, 17]. From a methodological perspective, the classic diffusion tensor imaging model is proving to be less important when compared with more advanced computational models for modelling diffusion in the intracellular and extracellular compartments [18]. The bi-tensor model that can be performed on singleshell diffusion scans yielding the free-water metric has shown considerable promise as a biomarker of progression in early stage PD [19]. There is also some evidence that examination of free-water in multiple nodes in the cerebellum and basal ganglia offer key insights into the degenerative patterns that may distinguish PD from other forms of Parkinsonism [20]. In addition, other models of diffusion that leverage multiple shells and more complex mathematics may also provide new paths to better understanding the tissue-specific changes in regions that include the substantia nigra but also nucleus basalis of Meynert, cerebellum, frontal cortex, brainstem, and many other structures [21]. Finally, although there is a major push to develop ligands for PET that will allow neuroimaging to directly assay alpha-synuclein, there is also a case to be made for developing diffusion or other structural imaging markers that may reflect alpha-synuclein pathology. This is because there could be considerable structural events (i.e., inflammation, neurodegeneration, gliosis) that occur prior to and after the clinical diagnosis of PD that are linked to alpha-synuclein pathology, and a multimodal approach will prove important in the future.

The final theme is in the area of clinical trials. At present, phase 1,2, and 3 clinical trials are not consistently using structural imaging read-outs for evaluation. It is the case though that many of the therapies under consideration are not directly affecting dopamine, and thus having non-invasive structural imaging assays ready for clinical trial use will prove important going forward [17].

\section{NUCLEAR IMAGING}

A major unsolved problem is the lack of a specific imaging marker for the pathological hallmark of
PD, alpha synuclein aggregates. There are a number of reasons for this. First, while many small aromatic molecules are known to bind to alphasynuclein fibrils, they all invariably bind with high affinity to beta amyloid which can also be present in elderly PD cases [22, 23]. Second, alpha-synuclein exists mainly in multiple oligomeric forms only some of which are toxic [24]. Small molecule ligands tend to bind weakly to these oligomers [25]. Third, alpha-synuclein is subject to multiple post translational modifications. While high throughput screening will no doubt continue to identify potential small molecule PET/SPECT ligands, an alternative way forward is to develop specific antibodies and peptides to alpha-synuclein oligomers and fibrils. This research is already in train, although a major problem is the transport of these ligands across the bloodbrain barrier (BBB). Peptides and vesicles can be tagged with moieties that bind to BBB transporters, such as transferrin [26]. However, extraction of these peptides is still slow relative to the short-lived PET and SPECT isotopes which have half-lives of minutes to hours. Other potential approaches may be to: (a) Design lipophilic cage compounds to passively transport peptide ligands [27]; (b) Use aptamers, which are circular loops of RNA that can be tailored to bind specific peptides and transport them across the BBB [28].

The pathology of PD leads to mitochondrial, lysosomal, and proteasomal dysfunction. Small molecule ligands such as enzyme inhibitors are now becoming available that could act as imaging biomarkers of these processes. BCPP-FE binds selectively to complex 1 in mitochondria and, in pharmacological doses acts to reduce mitochondrial membrane potential [29]. ${ }^{18}$ F-BCPP-FE has been shown to be a suitable agent for PET imaging in monkeys and is currently undergoing human trials. Agents that bind selectively to lysosomal and proteasomal components are also being screened as potential imaging markers given the association between glucocerebrosidase A deficiency and PD. One possible candidate could be the GBA inhibitor conduritol- $\beta$-epoxide (CBE).

Other fields of development in nuclear imaging include: (1) Surface receptor ligands which are sensitive to synaptic levels of transmitters. These already exist for dopamine $\left(11 \mathrm{C}\right.$-raclopride, ${ }^{11} \mathrm{C}$ FLB), serotonin ( $\left.{ }^{11} \mathrm{C}-\mathrm{AZ} 10419369\right)$, noradrenaline $\left({ }^{11} \mathrm{C}\right.$-yohimbine), and acetylcholine $\left({ }^{18} \mathrm{~F}\right.$-FP-TZTP $)$ but are inhibitors which are relatively insensitive to changes in transmitter fluxes. More sensitive, possibly agonist markers are required. Specific markers of 


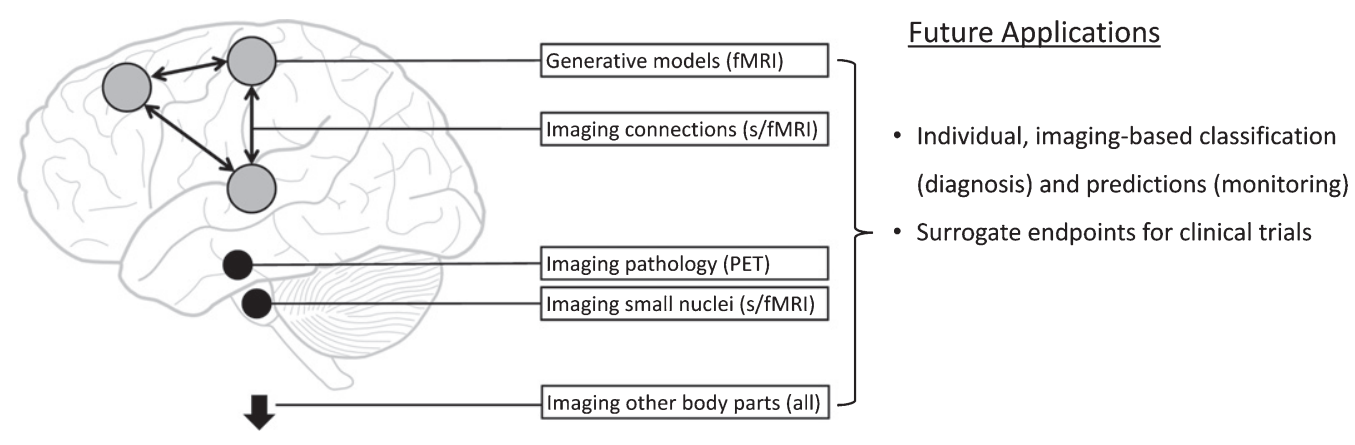

Fig. 1. Potential new imaging methods in Parkinson's disease. This figure schematically represents new approaches for functional magnetic resonance imaging (fMRI), structural MRI, and positron emission tomography (PET) imaging.

synaptic function, such as SV2A transporter ligands, are also under development [30]. ${ }^{11} \mathrm{C}-\mathrm{UCB}-\mathrm{J}$ is currently being trialled in $\mathrm{PD}$ cases with and without cognitive deficits. Finally, imaging peripheral systemic changes in PD has become of interest - it is known alpha-synuclein can be detected in skin and bowel and that sympathetic and parasympathetic activity can also be imaged. If a suitable alphasynuclein tracer emerges, then peripheral imaging may help to throw light on the proposal that transmission of alpha-synuclein aggregates starts peripherally and proceeds centrally.

\section{CONCLUSION}

Parkinson's disease is a brain disorder with distinct molecular, functional and structural features. These characteristics make it a prototypical neurological disorder where new neuroimaging techniques, especially the combination of multiple techniques in single patients [31], have the potential to make significant contributions to clinical practice (Fig. 1).

\section{ACKNOWLEDGMENTS}

The Parkinson Center of the Radboud university medical center was supported by a center of excellence grant of the Parkinson's Foundation. Rick Helmich was supported by grants from the Netherlands Organization for Scientific Research (VENI grant, \#91617077) and the Netherlands Organisation for Health Research and Development (OFF ROAD grant, \#91215208). David Vaillancourt was supported by the National Institutes of Health grants R01 NS052318 and U01 NS102038.

\section{CONFLICT OF INTEREST}

David Brooks is Consultant for GE Healthcare and Biogen. David Vaillancourt is co-founder and manager of Neuroimaging Solutions, LLC. Rick Helmich does not report conflicts of interest.

\section{REFERENCES}

[1] Helmich RC (2018) The cerebral basis of Parkinsonian tremor: A network perspective. Mov Disord 33, 219-231.

[2] Schindlbeck KA, Eidelberg D (2018) Network imaging biomarkers: Insights and clinical applications in Parkinson's disease. Lancet Neurol 17, 629-640.

[3] Kim J, Criaud M, Cho SS, Díez-Cirarda M, Mihaescu A, Coakeley S, Ghadery C, Valli M, Jacobs MF, Houle S, Strafella AP (2017) Abnormal intrinsic brain functional network dynamics in Parkinson's disease. Brain 140, 29552967.

[4] Stephan KE, Schlagenhauf F, Huys QJM, Raman S, Aponte EA, Brodersen KH, Rigoux L, Moran RJ, Daunizeau J, Dolan RJ, Friston KJ, Heinz A (2017) Computational neuroimaging strategies for single patient predictions. $\mathrm{Neu}$ roimage 145, 180-199.

[5] Dirkx MF, Ouden den HEM, Aarts E, Timmer MHM, Bloem BR, Toni I, Helmich RC (2017) Dopamine controls Parkinson's tremor by inhibiting the cerebellar thalamus. Brain 140, 721-734.

[6] Dirkx MF, Ouden den H, Aarts E, Timmer M, Bloem BR, Toni I, Helmich RC (2016) The cerebral network of Parkinson's tremor: An effective connectivity fMRI study. J Neurosci 36, 5362-5372.

[7] Rowe JB, Hughes LE, Barker RA, Owen AM (2010) Dynamic causal modelling of effective connectivity from fMRI: Are results reproducible and sensitive to Parkinson's disease and its treatment? Neuroimage 52, 1015-1026.

[8] Marquand AF, Haak KV, Beckmann CF (2017) Functional corticostriatal connection topographies predict goal-directed behaviour in humans. Nat Hum Behav 1, 0146.

[9] Helmich RC, Derikx LC, Bakker M, Scheeringa R, Bloem BR, Toni I (2010) Spatial remapping of cortico-striatal connectivity in Parkinson's disease. Cereb Cortex 20, 1175 1186.

[10] Marquand AF, Wolfers T, Mennes M, Buitelaar J, Beckmann CF (2016) Beyond lumping and splitting: A review of 
computational approaches for stratifying psychiatric disorders. Biol Psychiatry Cogn Neurosci Neuroimaging 1, 433-447.

[11] Lewis LD, Setsompop K, Rosen BR, Polimeni JR (2016) Fast fMRI can detect oscillatory neural activity in humans. Proc Natl Acad Sci U S A 113, E6679-E6685.

[12] Trapp C, Vakamudi K, Posse S (2018) On the detection of high frequency correlations in resting state fMRI. Neuroimage 164, 202-213.

[13] Lehéricy S, Vaillancourt DE, Seppi K, Monchi O, Rektorová I, Antonini A, McKeown MJ, Masellis M, Berg D, Rowe JB, Lewis SJG, Williams-Gray $\mathrm{CH}$, Tessitore A, Siebner HR, on behalf of the International Parkinson and Movement Disorder Society (IPMDS)-Neuroimaging Study Group (2017) The role of high-field magnetic resonance imaging in parkinsonian disorders: Pushing the boundaries forward. Mov Disord 27, 608-616.

[14] Visser E, Keuken MC, Forstmann BU, Jenkinson M (2016) Automated segmentation of the substantia nigra, subthalamic nucleus and red nucleus in $7 \mathrm{~T}$ data at young and old age. Neuroimage 139, 324-336.

[15] Lenglet C, Abosch A, Yacoub E, Martino FD, Sapiro G, Harel N (2012) Comprehensive in vivo mapping of the human basal ganglia and thalamic connectome in individuals using 7T MRI. PLoS ONE 7, e29153.

[16] Schulz J, Pagano G, Fernández Bonfante JA, Wilson H, Politis M (2018) Nucleus basalis of Meynert degeneration precedes and predicts cognitive impairment in Parkinson's disease. Brain 141, 1501-1516.

[17] Burciu RG, Ofori E, Archer DB, Wu SS, Pasternak O, McFarland NR, Okun MS, Vaillancourt DE (2017) Progression marker of Parkinson's disease: A 4-year multi-site imaging study. Brain 140, 2183-2192.

[18] Albi A, Pasternak O, Minati L, Marizzoni M, Bartrés-Faz D, Bargalló N, Bosch B, Rossini PM, Marra C, Müller B, Fiedler U, Wiltfang J, Roccatagliata L, Picco A, Nobili FM, Blin O, Sein J, Ranjeva J-P, Didic M, Bombois S, Lopes R, Bordet R, Gros-Dagnac H, Payoux P, Zoccatelli G, Alessandrini F, Beltramello A, Ferretti A, Caulo M, Aiello M, Cavaliere C, Soricelli A, Parnetti L, Tarducci R, Floridi P, Tsolaki M, Constantinidis M, Drevelegas A, Frisoni G, Jovicich J, The PharmaCog Consortium (2016) Free water elimination improves test-retest reproducibility of diffusion tensor imaging indices in the brain: A longitudinal multisite study of healthy elderly subjects. Hum Brain Mapp 38, 12-26.

[19] Yang J, Burciu RG, Vaillancourt DE (2018) Longitudinal progression markers of Parkinson's disease: Current view on structural imaging. Curr Neurol Neurosci Rep 18, 83.

[20] Planetta PJ, Ofori E, Pasternak O, Burciu RG, Shukla P, DeSimone JC, Okun MS, McFarland NR, Vaillancourt DE (2016) Free-water imaging in Parkinson's disease and atypical parkinsonism. Brain 139, 495-508.
[21] Zhang H, Schneider T, Wheeler-Kingshott CA, Alexander DC (2012) NODDI: Practical in vivo neurite orientation dispersion and density imaging of the human brain. $\mathrm{Neu}$ roimage $\mathbf{6 1}, 1000-1016$.

[22] Fodero-Tavoletti MT, Mulligan RS, Okamura N, Furumoto S, Rowe CC, Kudo Y, Masters CL, Cappai R, Yanai K, Villemagne VL (2009) In vitro characterisation of BF227 binding to $\alpha$-synuclein/Lewy bodies. Eur J Pharmacol 617, 54-58.

[23] Kotzbauer PT, Tu Z, Mach RH (2016) Current status of the development of PET radiotracers for imaging alpha synuclein aggregates in Lewy bodies and Lewy neurites. Clin Transl Imaging 5, 3-14.

[24] Shah M, Seibyl J, Cartier A, Bhatt R, Catafau AM (2014) Molecular imaging insights into neurodegeneration: Focus on $\alpha$-synuclein radiotracers. J Nucl Med 55, 1397-1400.

[25] Wagner J, Ryazanov S, Leonov A, Levin J, Shi S, Schmidt F, Prix C, Pan-Montojo F, Bertsch U, MittereggerKretzschmar G, Geissen M, Eiden M, Leidel F, Hirschberger T, Deeg AA, Krauth JJ, Zinth W, Tavan P, Pilger J, Zweckstetter M, Frank T, Bähr M, Weishaupt JH, Uhr M, Urlaub H, Teichmann U, Samwer M, Bötzel K, Groschup M, Kretzschmar H, Griesinger C, Giese A (2013) Anle138b: A novel oligomer modulator for disease-modifying therapy of neurodegenerative diseases such as prion and Parkinson's disease. Acta Neuropathol 125, 795-813.

[26] Johnsen KB, Burkhart A, Melander F, Kempen PJ, Vejlebo JB, Siupka P, Nielsen MS, Andresen TL, Moos T (2017) Targeting transferrin receptors at the blood-brain barrier improves the uptake of immunoliposomes and subsequent cargo transport into the brain parenchyma. Sci Rep 7, 10396.

[27] Zhang G, Mastalerz M (2014) Organic cage compounds - from shape-persistency to function. Chem Soc Rev 43, 1934-1947.

[28] Lakhin AV, Tarantul VZ, Gening LV (2013) Aptamers: Problems, solutions and prospects. Acta Naturae 5, 34-43.

[29] Kazami S, Nishiyama S, Kimura Y, Itoh H, Tsukada H (2018) BCPP compounds, PET probes for early therapeutic evaluations, specifically bind to mitochondrial complex I. Mitochondrion. doi: 10.1016/j.mito.2018.03.001

[30] Finnema SJ, Nabulsi NB, Mercier J, Lin S-F, Chen M-K, Matuskey D, Gallezot J-D, Henry S, Hannestad J, Huang Y, Carson RE (2018) Kinetic evaluation and test-retest reproducibility of [11C]UCB-J, a novel radioligand for positron emission tomography imaging of synaptic vesicle glycoprotein 2A in humans. J Cereb Blood Flow Metab 38, 2041-2052.

[31] Pyatigorskaya N, Magnin B, Mongin M, Yahia-Cherif L, Valabregue R, Arnaldi D, Ewenczyk C, Poupon C, Vidailhet M, Lehericy S (2018) Comparative Study of MRI Biomarkers in the Substantia Nigra to Discriminate Idiopathic Parkinson Disease. AJNR Am J Neuroradiol 39, 1460-1467. 